\title{
Recurrent Intracranial Meningeal Hemangiopericytoma with an Isolated Bone Metastasis: A Case Report and Literature Review
}

\author{
Görkem TÜRKKAN, ${ }^{1}$ Murat CALOĞLU, ${ }^{1}$ Ufuk USTA, ${ }^{2}$ Vuslat YÜRÜT CALOĞLU, ${ }^{1}$ \\ Hacı Mehmet SAYGI, ${ }^{3}$ Cem UZAL ${ }^{1}$ \\ ${ }^{1}$ Department of Radiation Oncology, Trakya University Faculty of Medicine, Edirne-Turkey \\ ${ }^{2}$ Department of Pathology, Trakya University Faculty of Medicine, Edirne-Turkey \\ ${ }^{3}$ Department of Neurosurgery, Trakya University Faculty of Medicine, Edirne-Turkey
}

\begin{abstract}
SUMMARY
Intracranial meningeal hemangiopericytoma is a rare vascular tumor with a high rate of both local recurrence and the development of distant metastases. The aim of this report is to present the first case of recurrent meningeal hemangiopericytoma with an isolated femur metastasis and discuss the clinical features and treatment of this rare tumor. A 46-year-old woman was admitted with the complaint of sudden loss of consciousness and subsequently underwent an urgent craniotomy with total excision of the lesion. The diagnosis was meningeal hemangiopericytoma. The patient presented with a painful left hip $5 \frac{1}{2}$ years after the initial surgery for the primary tumor. A lytic lesion, which was $4 \times 4.5 \mathrm{~cm}$ in diameter, was seen on an X-ray of the proximal left femur. The patient underwent lesion removal surgery and pathological diagnosis was compatible with meningeal hemangiopericytoma. At 69th month, no progression has been observed in follow-up. Long-term follow-up is mandatory for meningeal hemangiopericytoma due to high recurrence rate many years after diagnosis. Further multi-institutional studies are strongly needed to understand the clinical behavior and treatment modalities of this rare disease.

Keywords: Meningeal hemangiopericytoma; isolated metastasis; radiotherapy.

Copyright $\odot$ 2017, Turkish Society for Radiation Oncology
\end{abstract}

\section{Introduction}

Hemangiopericytomas are mesenchymal neoplasms originating from pericytes. Primary meningeal hemangiopericytomas are aggressive vascular tumors with a high rate of both local recurrence and the development of distant metastases.[1] They constitute approximately $2 \%$ of meningiomas and less than $1 \%$ of central nervous system (CNS) tumors. [2]

Most hemangiopericytomas are located in the musculoskeletal system and the skin. They are more common in men than in women. Intracranial hemangiopericytomas are rare and are often located supratentorially.[3] They are clinically and radiologically similar to meningiomas, but they have a stronger tendency for both local recurrence and the development of extracranial metastases than meningiomas.

The rates of metastatic disease vary from $23.4 \%[1]$ to $64 \%$. Metastases can be seen many years after removal of the primary tumor. The most common sites 


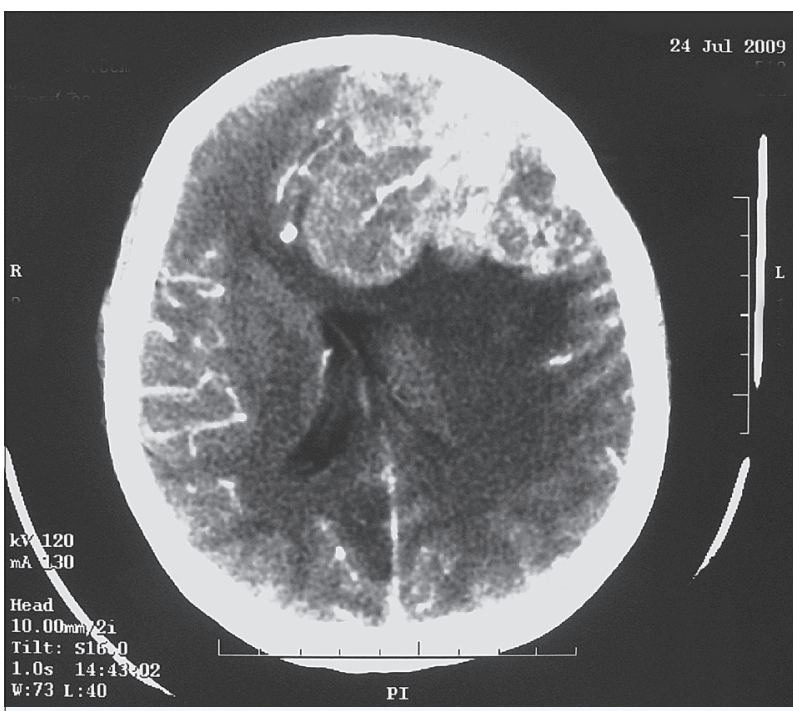

Fig. 1. Radiological images of the lesion. This figure shows a midline shift caused by a left frontotemporal lesion with perifocal oedema that contains calcifications in the axial slice of the CT scan.

of metastatic disease are the bone, liver, and lungs.[1] Most patients who develop extracranial metastases have multiple metastases in different sites.[4]

In this report, we present a rare case of an isolated metastatic recurrence of an intracranial meningeal hemangiopericytoma 5.5 years after the first diagnosis. In the medical literature, there are six reported cases of intracranial hemangiopericytomas with an isolated bone metastasis, and our case is the seventh. However, our patient is the first case with an isolated femur metastasis. The aim of this report is to present a very rare case and discuss the clinical features and treatment of this tumor.

\section{Case Report}

A 46-year-old woman was admitted to the emergency room in July 2009 with a complaint of sudden loss of consciousness. Computed tomography scan (CT) of the brain revealed a midline shift caused by a left frontotemporal lesion with a perifocal oedema that contained calcifications (Figure 1). On the basis of this imaging, a diagnosis of a meningioma was suspected. The patient underwent an urgent craniotomy with a total excision of the lesion.

Upon histopathological examination, microscopically, it was discovered that the whole specimen was composed of a tumor with extensively branched, thinwalled vessels. The tumor cells, which had enlarged
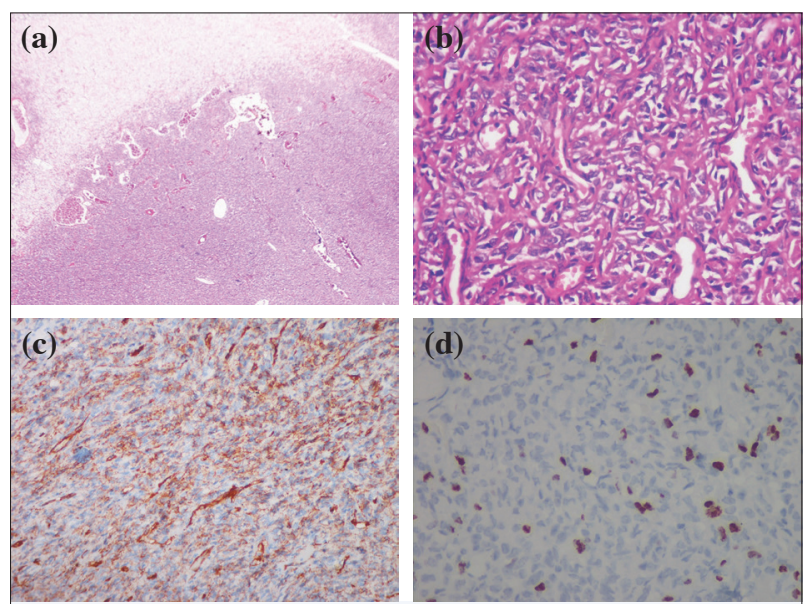

Fig. 2. Histopathological and immunohistochemical findings of the lesion. Panel a (HEx40) shows a panoramic view of the tumor with hemangiopericytomatous vasculature. Panel b (HEx400) shows moderate nuclear pleomorphism in the tumor cells around tiny branching vessels. Panel c (IHCx100) shows CD34 positivity in the tumor cells. Panel d (IHCx200) shows a high proliferation rate with $\mathrm{Ki}-67$.
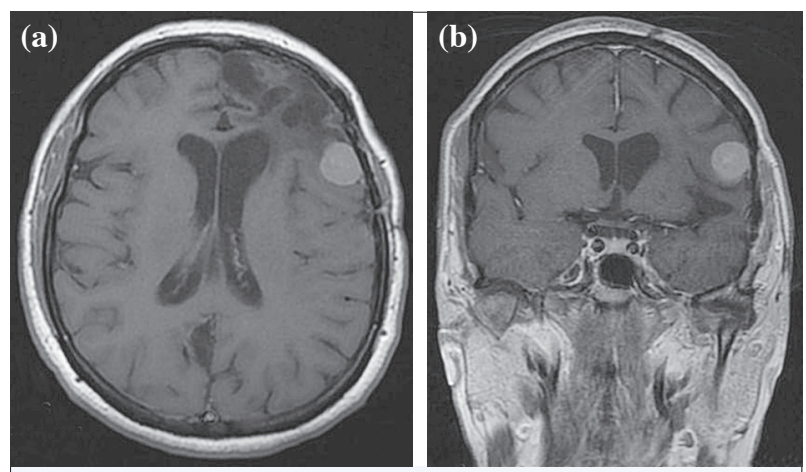

Fig. 3. MRI findings of the recurred lesion, which is 20 $\mathrm{mm}$ in diameter, next to the previous tumor site. Panel a shows an axial view of the initial tumor bed and the recurred lesion in the left frontal lobe. Panel $b$ shows a coronal view of the same lesion.

nuclei with moderate pleomorphism and scanty cytoplasm, were arranged as nested solid sheets around the vessels. Immunohistochemically, the tumor cells were positive for vimentin, CD34, and Bcl-2, but the cells were negative for CD31, desmin, pancytokeratin, S100 protein, Factor VIII, smooth muscle actin, and alpha epithelial membrane antigen. Ki-67 showed a proliferation in around $10 \%$ of tumor cells. The diagnosis of WHO Grade II hemangiopericytoma was accepted in accordance with these findings (Figure 2). 

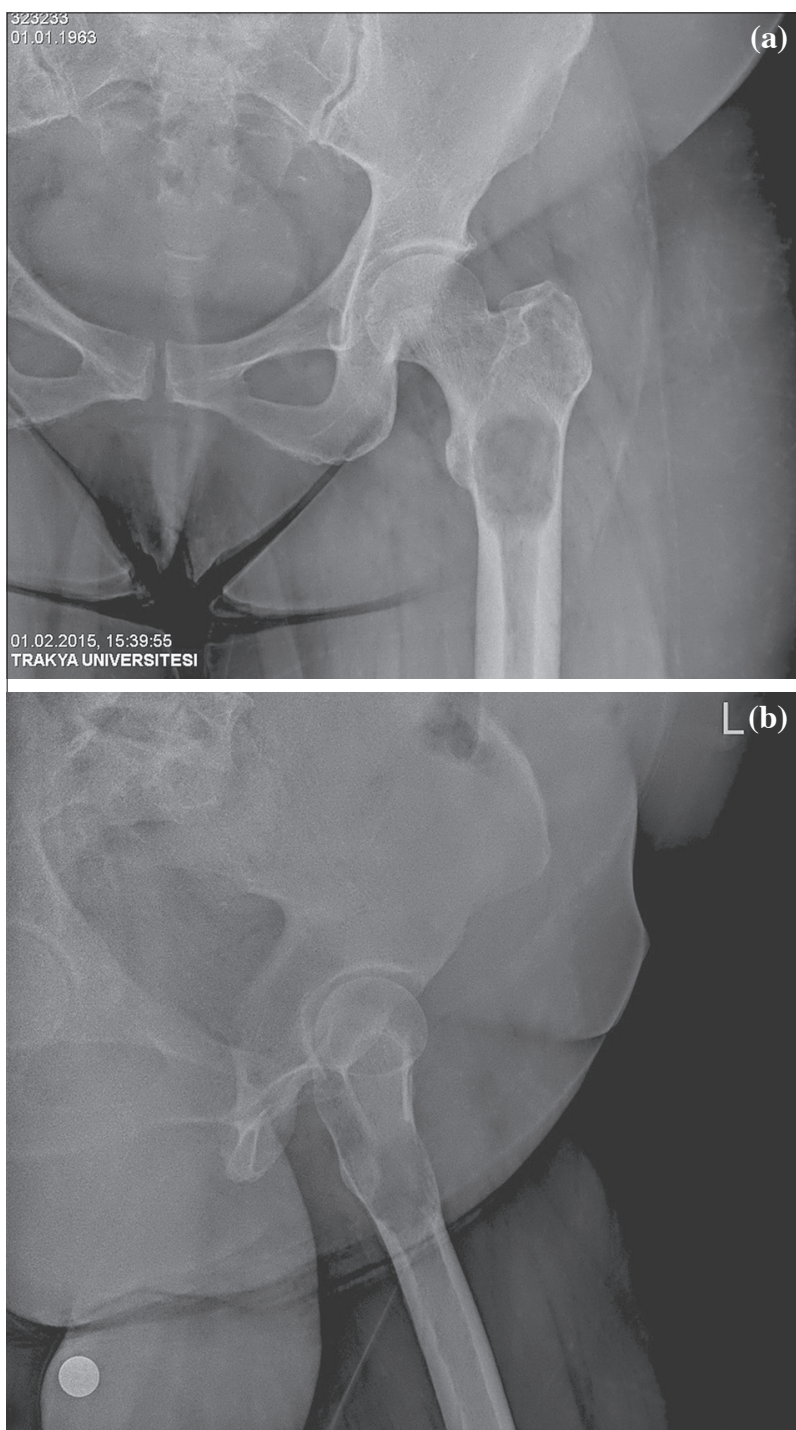

Fig. 4. X-ray images of the lytic metastasis on the femur neck on the level of the trochanter minor. The lesion destructed over $50 \%$ of bone diaphyseal cortices. Panel a shows an anterio-posterior view. Panel $b$ shows an oblique view of the left femur and the lesion.

No further treatment was offered by neurosurgeons and the patient attended follow-up visits. Radiological assessments with magnetic resonance imaging (MRI) of her brain showed no residual or recurrent tumor, and her neurological examinations were normal until 44 months after surgery.

In April 2013, the patient was asymptomatic, but a cranial MRI showed a lesion, which was $20 \mathrm{~mm}$ in diameter, in the left frontal lobe near the previous tumor site (Figure 3). Because of the local recurrence, the

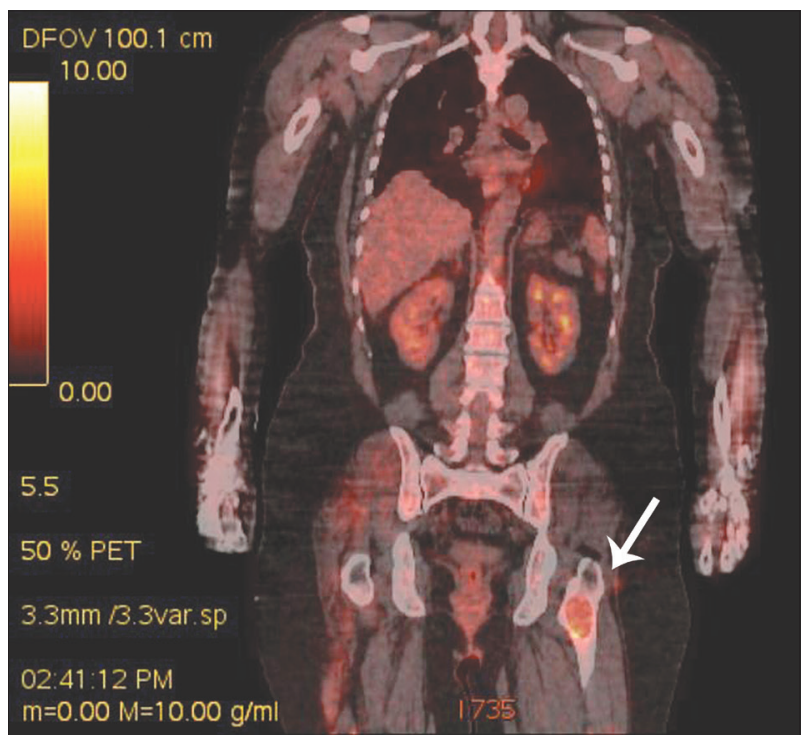

Fig. 5. PET-CT image of the patient showing isolated focal 18-FDG uptake in the trochanteric region of the left femur.

patient underwent another operation. The pathological diagnosis was compatible with the previous pathology (WHO Grade II hemangiopericytoma). The patient was referred to our clinic for adjuvant radiotherapy. There was no sign of a residual tumor on her cranial MRI, and 60 Gy (2 Gy/fraction daily and 5 fractions per week) of radiotherapy was postoperatively administered to the tumor bed.

During the first year after radiotherapy, the patient had no neurological symptoms, and radiologic followup was normal. One year after radiotherapy, she presented with a painful left hip. A lytic lesion, which was $4 \times 4.5 \mathrm{~cm}$ in diameter, was seen on an X-ray of the proximal left femur (Figure 4). A PET-CT performed for restaging and to find other possible metastatic lesions showed isolated focal 18-FDG uptake in the trochanteric region of the left femur on behalf of a malignant lesion that could have been metastatic or primary bone disease (Figure 5).

In February 2015, the patient underwent a resection of the left femur head, and prosthetic replacement surgery was performed. The pathological diagnosis was compatible with metastatic meningeal hemangiopericytoma (Figure 6). She was postoperatively given $30 \mathrm{~Gy}$ of radiotherapy for her metastatic lesion. The patient is attending follow-up visits. She is alive and well at 69 months after the initial diagnosis, and there has been no further progression of her disease. 


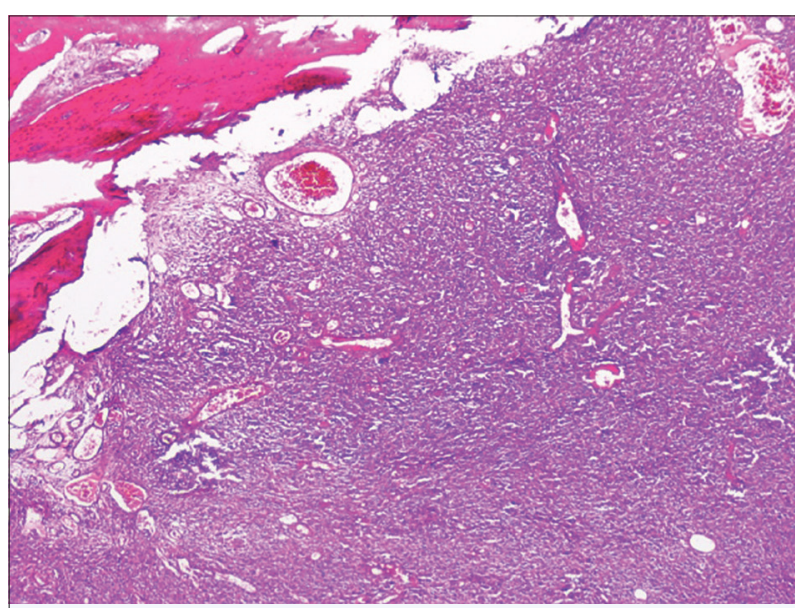

Fig. 6. (HEx40) Bone invasion of hemangiopericytoma (upper left).

Written informed consent was obtained from the patient who participated in this study.

\section{Discussion}

Hemangiopericytomas are rare vascular tumors arising from pericytes. They were first described by Murray and Stout in 1942.[3] Hemangiopericytomas mostly occur in the skin, retroperitoneal spaces, and the soft tissue of the lower extremities. However, they can rarely occur in the larynx, bones, spleen, and CNS as a primary tumor.[5] Intracranial meningeal hemangiopericytomas are uncommon. They account for approximately $2 \%$ of meningiomas and less than $1 \%$ of CNS tumors. [2]

Surgery is the mainstay of treatment for meningeal hemangiopericytomas. For unfavorably located tumors, conservative surgery with postoperative radiotherapy has been suggested. A dose of 50-60 Gy of radiotherapy is suggested to improve local control. The presented patient was treated and followed in surgical clinic after primary surgery. Since she was referred to our clinic after recurrence surgery, she has not received any radiotherapy. They have a strong tendency to recur locally and to develop distant metastases. Mena et al. reported that 57 patients developed local recurrence (60.6\%) and 22 patients developed single or multiple metastases (23.4\%) in their review, which consisted of 94 cases of CNS hemangiopericytoma.[1] The most common sites for metastases were the bone, liver, and lungs.[1]

In the literature review, we used PubMed to identify cases of bone metastases originating from intracranial meningeal hemangiopericytoma. The key words "hemangiopericytoma", "cranial", "meningeal", "bone", "metastasis", "radiotherapy", "surgery" were searched and cross-referenced. Articles including metastatic patients without bone metastasis were excluded. Twenty english-language articles were found, including $28 \mathrm{de}$ scribed cases of bone metastasis originating from an intracranial meningeal hemangiopericytoma. In three articles, the sites of the bone metastasis were not noted. Table 1 presents a summary of the reported cases. Of the 28 cases, 17 had bone metastasis, with the vertebra (61\%) being the most frequently involved site. Bone metastasis involved the pelvic bones in nine patients $(32 \%)$, the femur in seven patients $(25 \%)$, the ribs in four patients (14\%), the humerus in two patients (7\%), and the scapula in two patients (7\%). There are six reported cases with an isolated bone metastasis throughout the skeleton, but there are no cases with an isolated bone metastasis in the femur. Our patient is the first case with an isolated femur metastasis that originated from an intracranial meningeal hemangiopericytoma.

A bone metastasis can be seen as a lytic lesion with a sclerotic margin on plain roentgen film. On plain roentgen film and CT scans, the involved bones could show thinning and expansion.[6] The role of bone scans is limited for hemangiopericytomas because lesions may not be detected due to the lack of radionuclide isotope uptake of the metastases.

Metastases typically occur 63-99 months after the initial diagnosis of hemangiopericytomas. When a single bone metastasis is determined, synchronous multiple metastases can be observed in bones or in different organs, such as the lungs, liver, pancreas, and kidneys. The median survival rates of patients with metastases are paradoxically longer than expected. One study reported that the median survival rates of patients with and without systemic metastases were 202 months and 99 months, respectively.[7] High rates of local recurrences, the development of distant metastases, the occurrence of metastases a long period after the initial surgery, and the appearance of synchronous metastases are characteristic features of this tumor.[8]

Outcomes of chemotherapy for the management of metastatic disease, have been disappointing. The most frequent symptom at the time of diagnosis of bone metastases is pain. The total surgical excision of a bone metastasis may be beneficial for patients with limited distant disease, but the long-term benefits of this excision are unclear. Radiotherapy for bone metastases is effective for palliation. The most commonly used radiotherapy regimen is $30 \mathrm{~Gy} / 10 \mathrm{Fr}$. The aim of radiotherapy for bone metastases is not only pain relief but also 


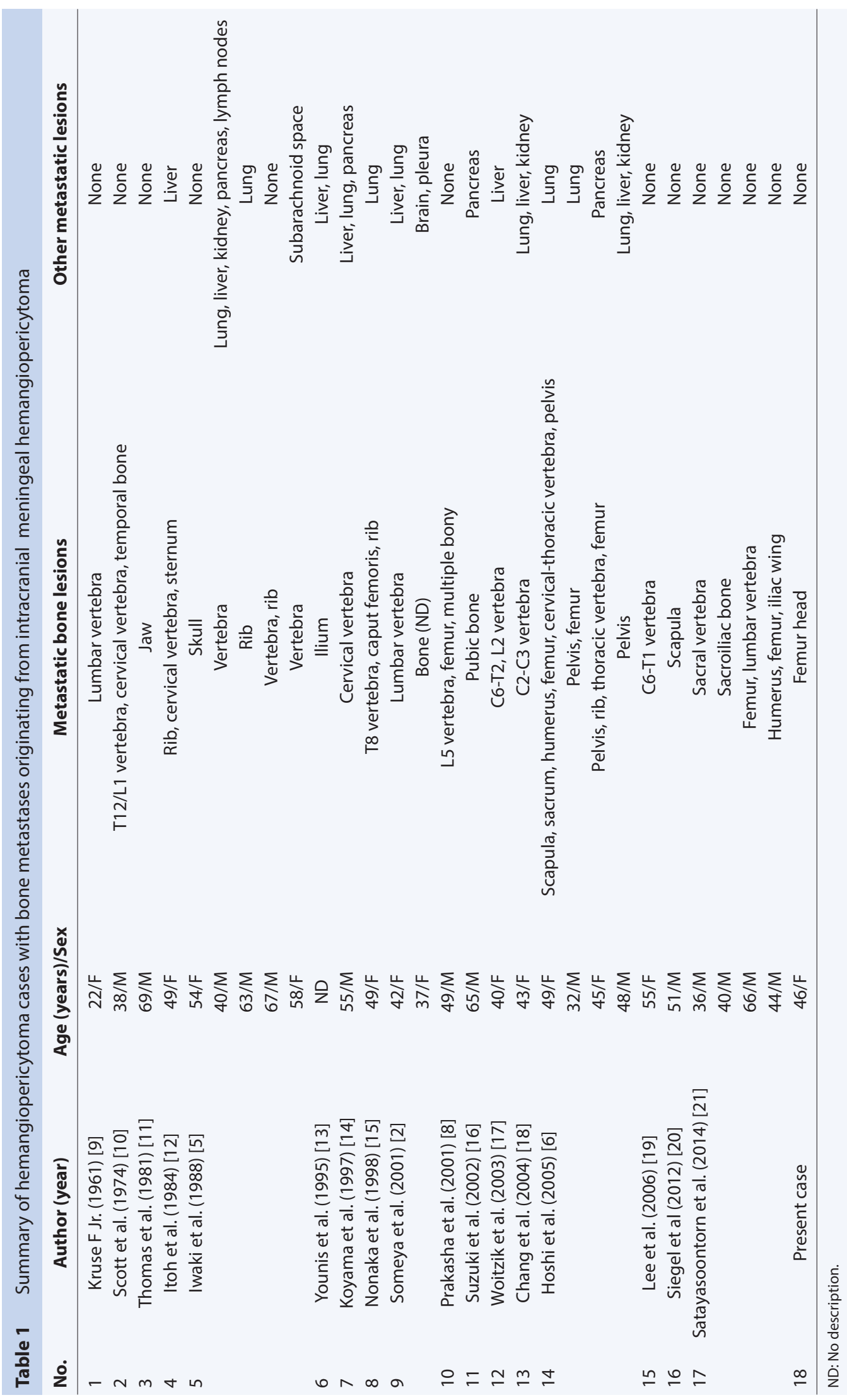


functional preservation and maintenance of a patient's quality of life. Besides, palliative radiotherapy is given as standart procedure to eradicate the microscopic tumor cells that may be left behind after metastasectomy. Consequently, 30 Gy (3 Gy/fraction daily and 5 fractions per week) of radiotherapy was given to metastasectomy area in concordance with the literature.

To the best of our knowledge, this report is the first reported case of an isolated femur metastasis that originated from an intracranial meningeal hemangiopericytoma 5.5 years after the initial surgery. The metastatic potential of this rare tumor should be considered. Long-term follow-up is mandatory due to the high recurrence rates many years after the surgical resection of the primary tumor. Since it is a rare disease, further multi-institutional studies are strongly needed to understand the clinical behavior and treatment modalities of this disease.

\section{Disclosure Statement}

The authors declare no conflicts of interest.

\section{References}

1. Mena H, Ribas JL, Pezeshkpour GH, Cowan DN, Parisi JE. Hemangiopericytoma of the central nervous system: a review of 94 cases. Hum Pathol 1991;22(1):8491.

2. Someya M, Sakata KI, Oouchi A, Nagakura H, Satoh M, Hareyama M. Four cases of meningeal hemangiopericytoma treated with surgery and radiotherapy. Jpn J Clin Oncol 2001;31(11):548-52.

3. Stout AP, Murray MR. Hemangiopericytoma: a vascular tumor featuring zimmermann's pericytes. Ann Surg 1942;116(1):26-33.

4. Ibis K, Saynak M, Yalta T, Ibis C, Kocak Z, Karadeniz A. Pancreas is an unusual initial metastatic site of intracranial hemangiopericytoma. Turk J Oncol 2016;31(2):55-60.

5. Iwaki T, Fukui M, Takeshita I, Tsuneyoshi M, Tateishi J. Hemangiopericytoma of the meninges: a clinicopathologic and immunohistochemical study. Clin Neuropathol 1988;7(3):93-9.

6. Hoshi M, Araki N, Naka N, Koizumi M, Hashimoto $\mathrm{N}$, Onishi $\mathrm{M}$, et al. Bone metastasis of intracranial meningeal hemangiopericytoma. Int J Clin Oncol 2005;10(3):208-13.

7. Han N, Kim H, Min SK, Paek SH, Park CK, Choi SH, et al. Meningeal Solitary Fibrous Tumors with Delayed Extracranial Metastasis. J Pathol Transl Med 2016;50(2):113-21.
8. Prakasha B, Jacob R, Dawson A, Joannides T. Haemangiopericytoma diagnosed from a metastasis 11years after surgery for "atypical meningioma". Br J Radiol 2001;74(885):856-8.

9. Kruse F. Hemangiopericytoma of the meniges (angioblastic meningioma of Cushing and Eisenhardt). Clinico-pathologic aspects and follow-up studies in 8 cases. Neurology 1961;11:771-7.

10. Scott M, Kellett G, Peale A. Angioblastic meningioma (hemangiopericytoma) of the cerebellar fossa with metastases to the temporal bone and the lumbar spine. Surg Neurol 1974;2(1):35-8.

11. Thomas HG, Dolman CL, Berry K. Malignant meningioma: clinical and pathological features. J Neurosurg 1981;55(6):929-34.

12. Itoh Y, Kowada M, Sakamoto T, Kojima H. Electron microscopic study of metastatic hemangiopericytic meningioma. [Article in Japanese] No Shinkei Geka 1984;12(10):1187-93. [Abstract]

13. Younis GA, Sawaya R, DeMonte F, Hess KR, Albrecht S, Bruner JM. Aggressive meningeal tumors: review of a series. J Neurosurg 1995;82(1):17-27.

14. Koyama H, Harada A, Nakao A, Nonami T, Kurokawa T, Kaneko T, et al. Intracranial hemangiopericytoma with metastasis to the pancreas. Case report and literature review. J Clin Gastroenterol 1997;25(4):706-8.

15. Nonaka M, Kohmura E, Hirata M, Hayakawa T. Metastatic meningeal hemangiopericytoma of thoracic spine. Clin Neurol Neurosurg 1998;100(3):228-30.

16. Suzuki H, Haga Y, Oguro K, Shinoda S, Masuzawa T, Kanai N. Intracranial hemangiopericytoma with extracranial metastasis occurring after 22 years. Neurol Med Chir (Tokyo) 2002;42(7):297-300.

17. Woitzik J, Sommer C, Krauss JK. Delayed manifestation of spinal metastasis: a special feature of hemangiopericytoma. Clin Neurol Neurosurg 2003;105(3):15966.

18. Chang CC, Chang YY, Lui CC, Huang CC, Liu JS. Meningeal hemangiopericytoma with delayed multiple distant metastases. J Chin Med Assoc 2004;67(10):52732.

19. Lee JK, Kim SH, Joo SP, Kim TS, Jung S, Kim JH, et al. Spinal metastasis from cranial meningeal hemangiopericytomas. Acta Neurochir (Wien) 2006;148(7):78790.

20. Siegel HJ, Lopez-Ben R, Sutton JH, Siegal GP. Intracranial meningeal hemangiopericytoma metastatic to the scapula. Orthopedics 2012;35(1):112-5.

21. Satayasoontorn K, Righi A, Gambarotti M, Merlino B, Brunocilla E, Vanel D. Meningeal hemangiopericytoma only diagnosed at the time of late bone metastasis. Skeletal Radiol 2014;43(11):1543-9. 